\title{
Study of Problems and Countermeasures on Ethical Review of Research in China
}

\author{
Xidong Deng ${ }^{1}$, Lingyan $\mathrm{He}^{2}$ \\ ${ }^{1}$ School of Economics and Management, China Three Gorges University, Yichang, China; ${ }^{2}$ School of Economics and Management, \\ China Three Gorges University, Yichang, China. \\ Email: 1067203988@qq.com, helingyanzi@163.com
}

Received July, 2013

\begin{abstract}
With the rapid development of biotechnology, ethical issues of scientific activities are more and more serious, while the ethical review as the main means of suppressing research ethics problems, it also has many problems in the process of practice. This article from the ethics review committee's organizational structure, operation specifications, capacity, the scope of review and external regulation summarize the main problems of China's research ethics review, and on this basis, we put forward the corresponding countermeasures, hoping to provide certain reference of our country's research ethics review practice, and to better safeguard the healthy development of our country's scientific research.
\end{abstract}

Keywords: Research Ethics; Ethical Review Committee; Ethical Review

\section{Introduction}

\subsection{The Necessity of Research Ethics Review}

With the rapid development and deepening of biotechnology, the contemporary scientific research is combined closely with commerce, market, financing funds, and the purpose and test pattern of the biomedical trials involving the human body has occurred and is undergoing a profound change, there are complex links between the various stakeholders, making scientific research process hide complex interst conflicts, many scientific research infringe upon the rights of the subjects, the project approval, design implementation, papers publishing, and the results reporting process filled with complex and sharp ethical issues. When scientific activities are no longer simply meaning the pursuit of objective knowledge, it is the value of a complex ethical connotation of social practice, and the value and purpose of human should be the inner dimension of scientific research, the necessity of introducing the ethical constrains into the scientific research will be understood[1].

To some extent, ethical review can keep scientific research objective, fair and according with ethical requirements. The ethical review of scientific research reflects on the respect and protection to the dignity, interests and rights of subjects, is a necessary step to promote scientific research to achieve scientific and ethical standards, playing a more and more important role for the healthy development of various research institutions. Ethical review can be strict to review process and be norms of scientific research from the origin of scientific research- project application process [2], so that the researchers will follow ethical principles in scientific research activities. Strictly controlling the test scheme and informed consent during the review process, ask them to put the subjects interests first, to maximize the protection of the rights of subjects. Above all, ethical review plays an important role and significant to the healthy development of our country's scientific research.

\subsection{The Necessity of Research Ethics Review}

Modern ethics review originated in Western, in 1946 "Nuremberg Code" make regulations for human medical trials to follow the ethical principles, and put forward four principles of human medical trials. In 1974 the U.S. Congress named the agency reviewing those research program involving human subjects Institutional Review Board, abbreviated as IRB[3]. Since then, the ethical review system has been running for more than thirty years in the West, and become the important measure to protect human research subjects.

Compared to Western countries, ethical review of our country have a late start, in the 1990s, to adapt the need to the rapid life development of biotechnology, in 1991 when carrying out a Sino-US cooperation in biomedical research project, in order to be able to continue to carry 
out research, at the request of the United States, we established the first ethical review committee. Subsequently technology and health authorities recognize the importance of ethical review, and then increasing the force of review biomedical research involving human research and application. In general terms, the construction of ethical review committee has experienced two stages: the first stage (about 1987 to 1997), during which the ethical review committee is mainly based on the hospital, and is named for hospital ethical committee, which is built in hospital, and staffed by a multidisciplinary medical ethical decision-making advisory organization, and the functions basically grouped into four categories: education and training, consulting coordination, supervisory review, ethics policy research, getting more attention to ethical review, and there are some initial translation, introduction and discussion, but not to involve ethical review of research on human issues widely; second phase (after 1997) mainly focuses on the growing number of ethics review committee for biomedical research involving human subjects and ethical review of pharmaceutical research, at the same time, the distribution of ethical review committee has also changed, the establishment not only in hospitals, and most medical schools, large medical research organizations have begun to focus on establishing ethics review committee to review the ethical issues of biomedical research, and some biological medical technology companies have also set up the organization.

Since our country introducing and learning ethics review regime has been twenty years, but the establishing and operating environment of ethical review regime in China is different from the ethical concept, theoretical foundation, cultural heritage and social environment in the West, and the working practice of ethical review committees around the country highlights the distinct characteristics with the West, a considerable part of the ethics review committee just threw a coat of ethical review, all kinds of about 500 ethics committee at all levels there is about only $20 \%$ with reasonable structure, the conditions and the ability to work independently, work procedures norms. Our ethics review system is imperfect: organizational construction and ethical review specific issues are increasingly exposed, and the validity of ethical review committees is eroded by many factors, so that the task of building ethics committee of standardization construction is urgent. In the context of the rapid development of biomedical research, how to be in line with international standards, build research ethics review system with Chinese characteristics and promote the healthy development of biomedical research, is both a practical problem needing to be solved and a theoretical problem needing in-depth study.

\section{The Analysis of Research Ethical Review Issues}

\subsection{Organization Building: Lack of Independence}

According to the experience of Western countries, the ethical review committee should be an independent organization, but in fact, in China's current ethical review system, none of our ethical review committee is completely independent, and they are affiliated with universities, research institutions, hospitals and health authorities ; ethical review activities can not be completely independent, researchers mostly from the unit, the agency with considerable academic authority makes the reviewer may be not made objective judgment. Meanwhile, one characteristic of the composition of our country's ethical review committee is that the government leaders of the institutions as the chairman or members of ethical review committee, because the clinical trial will bring a large number of research funding and reputation to research institutions, such as universities, hospitals and research institutions, so that the ethical review committee in protecting the interests of subjects will inevitably considering the interests of research institutions. The tension between the two tend weaken the insistence of the basic purpose of ethical review committee. It's usual to affect the objectivity and impartiality of the ethical review results because of taking the interests of institutions into consideration, and have to take into account the interests of both the subjects and the research institutions, which is conflict with the original intention of establishing ethical review committee. Such interest conflict could threaten the independence of ethical review. The review can not be in the proper review process, free from external influence, and in accordance with the ethics and the basic purpose, make independent judgment and decision, and then affecting the independence of ethical review.

\subsection{Operating Specifications: Lack of Standard Operating Procedures}

Due to ethical review committee in China starting relatively late, and imbalance development, the current standard and specifications of ethical review are still at the exploring stage. Ethical review committee generally follows the Declaration of Helsinki and other international norms, but such documents are demands principled and highly generalized, without detailed implementation specification for specific studies, and are difficult to grasp in practice. Laws and regulations of our country are made mainly according to the relevant principles of international guidelines, making no regulations or guidelines for specific content with procedural, operability and normative, making review decisions too subjective, dif- 
ferent ethical review committee with different review standards, the impartiality, authority and scientificity of the review be questioned.

\subsection{Members Capability: Lack of Continuing Education and Training}

Currently, the professionalism of members of ethical review committee and competence of ethical review work are not strong enough, and the ethical foundation of most research ethical committees is weak, lack of necessary knowledge and review capacity[4]. Although in recent years, the training program of research ethics keeps increasing, it's still far to meet actual needs. The ethical review committee itself is lacking of resources to provide training, so members have no way to get education. This greatly limits the ethical review committee to improve its own review capabilities.

\subsection{Review Scope: Lack of Feedback and Track- ing Review}

Most review of ethical review committee is limited to the initial research programs, and after pass the review and enter through the pilot phase, the ethical review committee is seldom to do tracking review and oversight for specific implementation process, informed consent process and the protection of the real situation of the subjects, and even lack of necessary attention and tracking review for the changes, modifications and other issues of informed consent of research projects, and rarely asking researchers to submit the changes and other relevant feedback information[5]. Meanwhile, after raising the questions in the tracking, it's always lacking of the tracking review for problem-solving situations, making some problems found can not really be solved. This has resulted in ethical review committee not clearly understanding the real situation of informed consent and the protection of the subjects, to a certain extent, making ethical review as empty shells.

\subsection{External Oversight: Lack of Effective Supervision and Management Mechanism}

In other countries, the regulatory model for ethical safeguards has attracted a lot of attention: ethical review has evolved into a "research ethics industry." Western countries with complete ethics review system all have a specialized agency to oversee the operation of ethical review committee, such as the U.S. OHRP, UK NRES and the Swedish central ethical review committee. In our country, the ethical review committee is mainly managed by the Ministry of Health and the Food and Drug Administration at the macro level, and without a regulatory ethics review committee. Compared with foreign countries, in our country ethical review committee has neither formed external supervision mechanism to ethical review committee, nor formed a self-assessment procedure. We lack specialized agencies to evaluate and assess the situation of the implementation of ethical review, ensure the committee to act by chapter, and punish irregularities.

\section{Countermeasures and Suggestions}

\subsection{To Strengthen the Construction of Certified System of Ethical Review Committee}

The low threshold to establish ethics committee is one of the roots resulting in uneven quality of each ethical review committee [5]. To improve the access threshold and practice quality, creating a third-party certification assessment sys-tem is one of important measure to further improve the capacity and quality of ethical review committee. To move in the direction to establish a professional focus in order to ensure ethical review to operate healthy in tracks of high efficiency and high quality, will be more conducive to the major ethical issues about subjects protection with enough voice and execution. It requires specification of barriers to entry and continuously evaluation of their ability to practice, eliminating those committees undertaking fewer projects, weak capacity to review. Currently a number of ethical review committees invite foreign counterparts or institutions to help improve ethical review capacity, which indicates that our country have urgent desire and pursuit to improve the capacity for ethical review and management, and it's urgent to set up a ethical review evaluation criteria system combined with international standards and appropriate to the cultural, economic, racial, religious characteristics.

\subsection{To Establish Standard Operating Procedures}

Whether we can fulfill the review functions with high efficiency and high standards and ensure the safety and interests of subjects, depends on the efficient management system and operational standard operating procedures. Therefore, in order to improve the quality of ethical review committees, ethics committees should have a complete organization and system, should develop comprehensive censorship and procedures, establish standard operating procedures SOP. Based on both inside and outside relevant laws and regulations, combining with the situation of research and project review, pre-practical experience of the ethical review committee, and drawing on foreign experience, design the SOP for local ethical committee system to control the quality of ethical review. But it's important to note that only the most optimal design through constant practice, could be realized under current, specific conditions can be called standard operating procedures, we should focus on operability and accuracy. 
In practice, standard operating procedures can be divided into the following seven sections: the design of standard operating rules and guidelines; the structure of the ethical review committee; the ways and category of ethical review; ethical review processes; various ethical review processes; office management, including communication records; document and file management.

\subsection{To Strengthen the Training and Continuing Education for Members}

With the development of scientific research, the ways and methods of ethical review are constantly updated, regular, continuous and standardized training for ethical knowledge has a crucial role in comprehensively improving the professional level for the ethical committee members to carry out the ethical review[6]. Through training, on a understanding level further improve the ethical review staff's understanding to the protection of subjects' interests on human test, and be clear to the the necessity and importance of ethical review; On a technical level regulate the process of ethical review, enhance the professional knowledge and skills of ethical review ; improve the quality of ethical review, improve management capacity, improve document and file management to ensure the quality of ethical review.

Therefore, ethical review committee needs to take full advantage of existing resources, through various forms to train for members. Relying on relevant academic institutions, medical schools and medical technology groups, etc., organization of training courses, conferences and seminars, train on the ability of to review and relevant laws and regulations for members, and strengthen the understanding of review process, review highlights to improve the skills of ethical review committee. In addition, we should strengthen exchanges, build homes interpersonal, inter-provincial and international learning exchange platform for the exchange of learning and training. Establish a standard research ethics continuing education system, the level of professional ability as the key factor to assess each agency. We may consider to establish qualification authentication mechanism for members, those who have the appropriate ability could be in, thereby improving the professionalism and effectiveness of ethical review overall.

\subsection{To Establish the Feedback and Tracking Review Mechanisms}

An ethical review, the ethical review of the clinical investigation is not just limited to the examination before the study began, but also including the tracking review after the study began, including review, repair positive case review, annual/regular tracking review, serious adverse events review, contrary to program review, review of early termination, concluding review. Tracking review of an ethics review is an important part of ethical review, to some extent, its practical significance is even beyond the ethical review itself. Tracking review is a research project within the framework of the ethical review implementation through effective safeguards, can ensure the implementation of research projects accord with the approved plan, is the core means to ensure subjects' interests, to protect medical research and scientific credibility[7], to enhance the quality of tracking the review, it is necessary to strengthen the ethical review committee of supervision and management. In the tracking review process, taking into account the report of the researchers active inherent defect, should subjects feedback deserves attention.

Our country can learn from the U.S. experience, requiring a research project on human trials to determine the person responsible for understanding the subject concerns, answering questions and record before the application submitted to tests, and build a scientific feedback mechanism to solve the feedback problem of subjects reasonably.

\subsection{To Speed up the Construction of Regulatory System}

The key of ethical review is to establish ethics review system, build ethical review committees, and implement the ethics review. The ability of ethical review commit-tee determines the construction level of the ethical re-view platform, but the construction of ethical review platform must not be limited to set up a ethical commit-tee, the smooth operation need for a comprehensive operation supervision system as a guarantee[8]. The external regulatory and its own review ability of ethical review committee are complementary and mutually reinforcing. To improve our current ethical review committee management mechanism, need to establish a special institution independent of the Drug Administration, Ministry of Scientific Research and the Ministry of Health responsible for management and supervision for the ethical re-view committee, and gradually establish more comprehensive regulatory system to carry out quality assessment and quality promotion project. This is one of the most important direction for the ethical review committee improving and developing itself. Based on the international ethical review, supervision and evaluation standard, en-sure the authority, independence, objectivity and impartiality of ethical review, to avoid the injustice resulting from the interests confilt between various institutions. The content of the regulation for ethical review committee is of multifarious, we should determine the content of regulation based on the main problems ethical review committee facing to, including qualification, standard operating procedures, ethical independence, 
tracking review, conflict of interests, training and the availability of education etc.; We need to gradually increase supervision in these aspects, to realize the good interactive development between external supervision and internal review ability .

Specifically, first, refine the regulatory approach; secondly, require ethical review committees to make a decision in writing in order to prepare for post-review, and require researchers to submit a written report to the ethical review committee to protect the rights of subjects better; last, if regulators find problems of ethical review committee, shall order it to make corrections, if it dam-ages to the rights of subjects, should notify aborting human medical trials; if find serious problems, should have a deadline for correction, if not, should suspend its ethical review qualifications[9].

\section{Summary}

Social's progress and development is inseparable from scientific research, but research activities involved in a lot of hidden ethical issues because of the conflict of interests among all stakeholders. Ethical review committee promotes research activities to develop healthily and normatively, but it is restricted by various factors in the process of practice, thus affect the quality of ethical review. It requires that we comply with international norms and shared ethical guidelines combined with domestic laws and regulations, through the construction of ethical review board-certified system, establish and implement standard operating procedures of the ethical review committee, and strengthen the training and continuing education for members, establish feedback and tracking mechanisms, accelerate the monitoring system to improve the quality of ethical review, quality improvement will be more conducive to promote the common devel- opment of ethical review committees, and then protect the healthy development of research activities.

\section{REFERENCES}

[1] W. W. Duan, "Research Ethics and Imformation Rights,” 2007, p. 003.

[2] M. Shi, J. Y. Li and T. Wu, "The Practice and Experience of the Standardized Operation in Ethical Review in $\mathrm{Hu}-$ man Involved Biomedical Research,” Medicine and Philosophy (Humanisitic \& Social Medicine Edition), Vol. 08, 2009, pp. 16-17.

[3] R. Deng, "Ethics Review of Scientific Research in China----History, Status and Reflection,” Studies in Dialectics of Nature, Vol. 08, 2011, pp. 116-121.

[4] Z. G. Dan, J. Yu and Z. H. Han, "Research Ethics Review for the Promotion of Hospital Scientific Research Management," Chinese Journal of Misdiagnostics, Vol. 34, 2010, pp. 8320-8321.

[5] J. Huang, J. H. Hu, J. Pu and Y. J. Xiang, "Standardization Construction and Quality Improvement Strategies of Ethical Review Committee,” Chinese Hospital Management, Vol. 12, 2012, pp. 20-21.

[6] X. D. Zheng, B. Jiang, W. Lu, Y. L. Sun and J. He, "Medical Ethical: Problems in Surveillance," Academic Journal of Second Military Medical University, Vol. 06, 2007, pp. 667-669.

[7] X. Zhang, M. Yin and D. R. Liu, "Exploration and Analysis of Tracking Review by Ethics Review Committee," Medicin and Philosophy, Vol. 04, 2012, pp. 26-27+51.

[8] J. Z. Liu, Y. C. Tu and X. Zhang, "Discussion on Construction of Ethics Review Platform for Traditional Chinese Medicine Clinical Researches," World Chinese Medicine, Vol. 02, 2013, pp. 209-211.

[9] W. Y. Gong, "Research on Legal Problems of Human Medical Experiments of our Country," Tian Jin University, 2012. 